\title{
EDITORIAL
}

\section{A COVID-19 SUPPORT PLATFORM FOR LONG TERM CARE FACILITIES}

\author{
Y. ROLLAND ${ }^{1}$, A. BENETOS ${ }^{2}$, H. VILLARS ${ }^{1}$, H. BRAUN ${ }^{1}$, H. BLAIN ${ }^{3}$

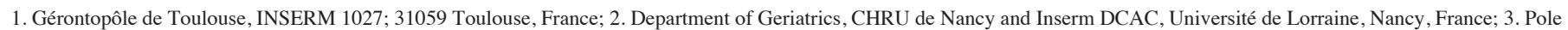

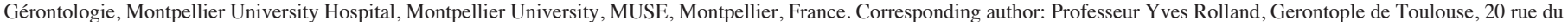 \\ Pont Saint Pierre, 31059 Toulouse, France, Tel: 05617764 45, E-Mail : rolland.y@chu-toulouse.fr
}

"We are at war», said President Emmanuel Macron on 16th March 2020, about the health crisis against COVID-19, then in full epidemic expansion in France. Nearly 600,000 people ( $6 \%$ of people aged 85 or older) live in the 7,200 French nursing homes. In addition to the now well-known risk factors of age, dependence and associated co-morbidities, community life exposes NH residents to specific increased risks of this easily inter human transmissible virus (1). NH's residents are particularly at risk and may pay a heavy price during this epidemic. In the case study described recently by McMichael et al. in Washington, 19 days after a first case of Covid-19 diagnosed in a resident of a skilled long-term care facility, 167 confirmed cases of Covid-19 affecting 101 residents, 50 health care personnel, and 16 visitors were found to be epidemiologically linked, showing the possible quick dissemination of the epidemic in nursing homes. Thus, the death rate of residents with confirmed Covid-10 was 33.7\%, testifying the threat of this pathology in $\mathrm{NH}$ population (3). Based on these findings and many others in the world, it appears clearly that implementing an effective prevention and care strategy in $\mathrm{NH}$ is very urgent. More than ever, innovation in NH must be implemented. The strategy put in place between the geriatrics department and $\mathrm{NH}$ in in the Occitanie Region (South of France) to fight this unprecedented threat could inspire other regions and countries facing the COVID-19 epidemic.

Beyond the strict application of all barrier protection gestures, the prohibition of visits so that only caregivers, wearing masks are in contact with residents, it became clear that the threat justified a larger and more specific strategy. The hospital medical/nurses consultation activities, reduced during the epidemic period, have been redeployed to a team specifically dedicated to support nursing home staff and resident during this epidemic period. This hospital COVID-19 support platform for $\mathrm{NH}$, was quickly implemented thanks to an old partnership between hospital geriatric units and NH whether public or private.

Objectives of the COVID-19 support platform for NH were to respond to the information needs of $\mathrm{NH}$ staff, - to trigger the carrying out of Covid-19 biological test in suspected residents and caregivers, - to advise and support NH by telemedicine, to help decision-making process to the sector and to specific care, in particular by supporting ethical and collegial reflection around the care of positive Covid-19 residents, - helping to protect NH staff, and - provide information on care procedures.

For the prevention and anticipation of an epidemic situation in the NH, the COVID-19 support platform for $\mathrm{NH}$ has first set up a hotline open 7 days a week to train and answer the questions of the coordinating doctors. In France, residents live in $\mathrm{NH}$ when home support is no longer possible and usually until their death. Residents are over 86 years of age. The annual death rate is often over $30 \%$. Each registered $\mathrm{NH}$ is required to have a coordinating physician among its staff members. This coordinating physician is a medical physician who has received at least a 140-hour geriatric training (70 hours of theoretical and 70 hours of practical courses). He is responsible for the comprehensive geriatric assessment of each $\mathrm{NH}$ resident and for the $\mathrm{NH}$ healthcare coordination. However, treatments including drug prescription or decision of hospitalisation remain usually under the responsibility of the resident's general practitioner. The risk of contamination of the residents being dependent on the contamination of the caregivers, the visits within the NHs of the general practitioners, themselves in contact with patients affected by Covid-19 in their activity in the community, was limited to the maximum. This situation forced the coordinating doctors to take the lead in care during the crisis. The hotline consisted of a unique telephone number, widely distributed to the association of coordinating physicians and $\mathrm{NH}$ mailing list. It allows direct contact with a senior geriatrician from the geriatric hospital department. The hotline is also based on a website (https://covid19.rehpa.org/) allowing the posting of documents necessary for the management of the crisis. A list of tasks (to do list for the coordinating physician) to prevent and be ready for an epidemic situation in $\mathrm{NH}$ is proposed.

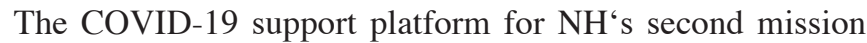
is to screen for COVID-19 in the NH. A mobile team dedicated to Covid-19 samples has been formed. It operates from 9 am to 7 pm, 7 days a week. Two volunteers geriatric nurses carry out COVID-19 tests by chain amplification by reverse transcriptase polymerase in real time on the nasopharyngeal swab, at the request of the telephone platform. Since the start of the crisis, the mobile sampling team has been mobilized 2 to 6 times a day. The requests relate to suspected COVID-19 infection in residents and caregivers. The screening and eviction of infected and potentially contaminating caregivers is a key mission of this team. Indeed, since the ban on family visits 2 weeks ago, only the healthcare team can be contaminating for residents. 
The visit of the team is also an opportunity to reinforce the barrier measurement messages and in particular the use of the mask among caregivers.

The third mission of the COVID-19 support platform for $\mathrm{NH}$ is to help the NH care staff to manage the crisis of a COVID epidemic in NH. This aid is organized from telemedicine consultation. Geriatric hospital expertise, in palliative care, in ethics, in management of behavioral disorders such as wandering in the context of COVID infection is mobilized. The knowledge of the care sector by the geriatric team makes it possible to consider resorting to hospitalization if needed, or using palliative care networks in the $\mathrm{NH}$, home hospitalization resources, procedure of care, or collegial decision. The strategy to limit the spread of the epidemic is discussed with the $\mathrm{NH}$ team. In a complex situation, the mobile geriatric team of the hospital may be called upon to move to the NH.

Fifteen days after the instructions to contain the population, it is not the hour of fatalism in NH. Despite the consequences on their hospital care unit, it is the duty of hospital geriatricians to be fully mobilized with the actors of care in $\mathrm{NH}$ and do everything possible to limit the dreaded consequences of the epidemic. The strategy that we have implemented in the French Occitanie region is currently being deployed at the national level. The development of a national register of deaths in $\mathrm{NH}$ will make it possible to judge the effects of the actions carried out. This strategy must be adjusted according to the human resources of each geriatric hospital department. Certain initiatives relating to the sampling strategies of residents and caregivers will have to be analyzed as soon as possible in order to perfect the measures in progress.

Disclosure: The authors have no conflicts of interest.

\section{References}

1. Cesari M, Geriatric medicine in Italy in the time of COVID-19. J Nutr Health Aging. 2020; 24(5): 459-460

2. McMichael TM, Currie DW, Clark S, Pogosjans S, Kay M, Schwartz NG, et al. Epidemiology of Covid-19 in a Long-Term Care Facility in King County, Washington. N Engl J Med. 2020 Mar 27.

3. Landi F, The new challenge of geriatrics: Saving frail older people from the SARSCOV-2 Pandemic Infection. J Nutr Health Aging. 2020; 24(5): 466-470 